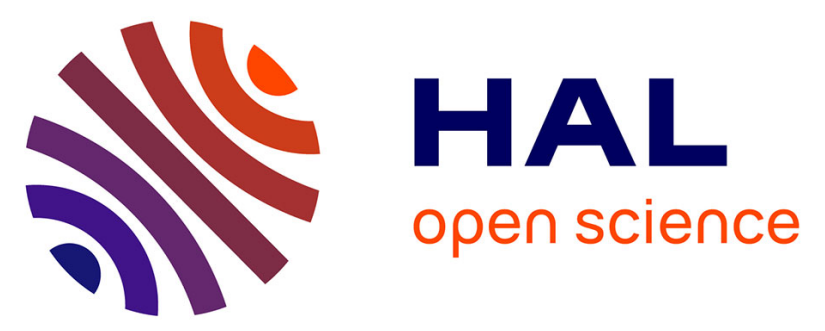

\title{
PROPRIÉTÉS THERMIQUES MÉCANIQUES OPTIQUES ET ÉLECTRIQUESANOMALIE DE CHALEUR SPÉCIFIQUE AU VOISINAGE DES TRANSITIONS FERROPARAÉLECTRIQUES
}

\author{
M. C. Bernard, J. Perrigot, Murielle Richard, L. Eyraud
}

\section{To cite this version:}

M. C. Bernard, J. Perrigot, Murielle Richard, L. Eyraud. PROPRIÉTÉS THERMIQUES MÉCANIQUES OPTIQUES ET ÉLECTRIQUESANOMALIE DE CHALEUR SPÉCIFIQUE AU VOISINAGE DES TRANSITIONS FERROPARAÉLECTRIQUES. Journal de Physique Colloques, 1972, 33 (C2), pp.C2-93-C2-94. 10.1051/jphyscol:1972228 . jpa-00214964

HAL Id: jpa-00214964 https://hal.science/jpa-00214964

Submitted on 1 Jan 1972

HAL is a multi-disciplinary open access archive for the deposit and dissemination of scientific research documents, whether they are published or not. The documents may come from teaching and research institutions in France or abroad, or from public or private research centers.
L'archive ouverte pluridisciplinaire HAL, est destinée au dépôt et à la diffusion de documents scientifiques de niveau recherche, publiés ou non, émanant des établissements d'enseignement et de recherche français ou étrangers, des laboratoires publics ou privés. 


\title{
PROPRIETEES THERMIQUES MÉCANIQUES OPTIQUES ET ÉLECTRIQUES
}

\section{ANOMALIE DE CHALEUR SPÉCIFIQUE AU VOISINAGE DES TRANSITIONS FERROPARAÉLECTRIQUES}

\author{
M. BERNARD, J. PERRIGOT, M. RICHARD et L. EYRAUD \\ Laboratoire de Ferroélectricité, INSA, \\ 20, avenue Albert-Einstein, 69, Villeurbanne, France
}

\begin{abstract}
Résumé. - Nous donnons des courbes de variation de chaleur spéctfique en fonction de la température au voisinage de transitions ferro paraélectriques. Deux exemples caractéristiques ont été choisis: le T. G. S. pour les transitions du $2^{e}$ ordre et $\mathrm{PbTiO}_{3}$ pour celles du premier ordre. L'existence d'une hystérésis thermique dans le second cas est mis à profit pour déterminer la chaleur latente associée à la transition.
\end{abstract}

On sait que lorsqu'on élève la température d'un matériau ferroélectrique ou antiferroélectrique, il se produit un effet endothermique important au passage du corps dans l'état paraélectrique [1], [2]. Cet effet qui devient exothermique en descente de température couvre généralement une gamme de température qui peut varier de quelques degrés à plusieurs dizaines de degrés Celsius suivant la nature du matériau étudié. Les contributions majeures à ce phénomène sont: l'effet calorifique résultant de la variation de la polarisation spontanée ; celui engendré par les contractions ou dilatations de la maille cristalline; celui lié à la disparition ou l'apparition d'une structure de domaine... [3], [4].

Un problème important concernant ces transitions est de savoir si du point de vue de la thermodynamique et suivant les corps étudiés, elles sont du premier ou du deuxième ordre. En d'autres termes, l'effet calorifique précédent résulte-t-il seulement d'une variation de la chaleur spécifique ou existe-t-il une chaleur latente associée à la transition ? La réponse à cette interrogation est simple dans le cas où le matériau étudié est un monocristal ayant une température de Curie bien définie. Par exemple, pour les cristaux de T. G. S. on constate une augmentation progressive de la chaleur spécifique jusqu'à la température de Curie $T_{\mathfrak{c}}=49^{\circ} \mathrm{C}$, suivie d'une chute rapide jusqu'à environ $53^{\circ} \mathrm{C}$; ensuite la valeur de $C_{\mathrm{p}}$ teprend son ascension régulière (Fig. 1). L'absence de valeur anormalement élevée de la chaleur spécifique au voisinage de $T_{\mathrm{c}}$ permet de conclure qu'il s'agit d'une transition du deuxième ordre.

Par contre, quand on étudie des échantillons céramiques frittés de titanate de plomb par exemple il existe à l'intérieur du matériau des contraintes qui engendrent un étalement de la transition sur plusieurs degrés et il est alors très difficile de distinguer une chaleur latente d'une variation progressive de chaleur

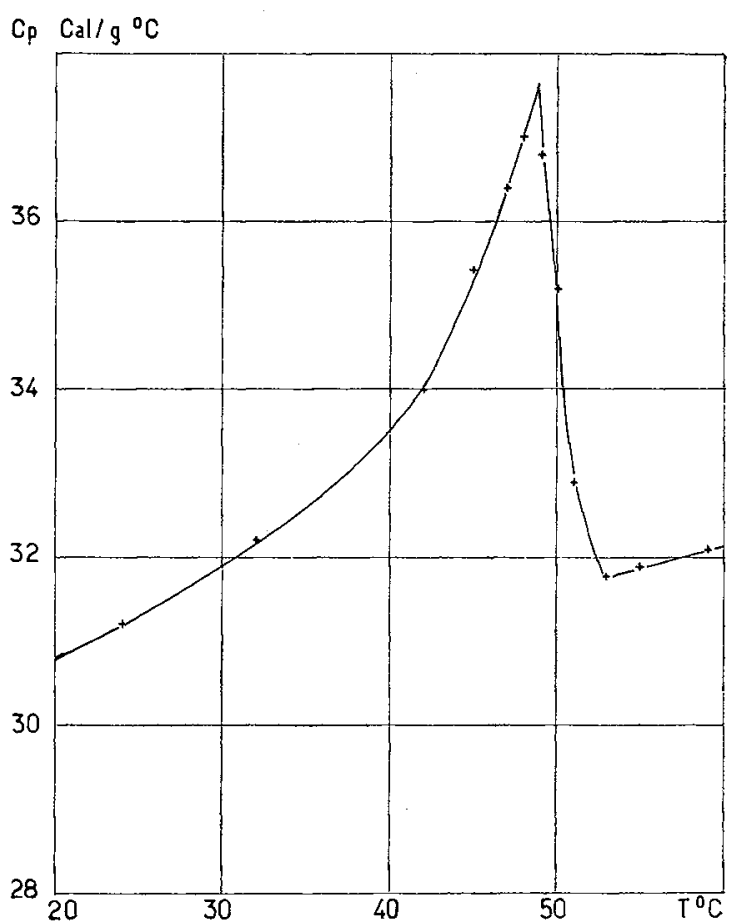

FIG. 1. - Chaleur spécifique du T. G. S.

spécifique. Cependant, il nous a été possible en utilisant un calorimètre isotherme à chute [5], [6] de donner une réponse à cette question.

Cet appareil nous permet de soumettre l'échantillon à des créneaux de température de hauteur $\Delta T$ au voisinage de $T_{\mathrm{c}}$. On peut alors mesurer $C_{\mathrm{p}}=\Delta W / \Delta T$ pour des $\Delta T$ positifs ou négatifs l'amplitude est de 1 à $2^{\circ} \mathrm{C}$. On peut alors constater qu'en montée de température et juste avant de franchir le point de Curie, la chaleur spécifique mesurée lorsqu'on passe de la température $T_{1}$ à la température $T_{2}$ soit 
$C_{\mathrm{p}_{1}}=\Delta W_{1} /\left(T_{2}-T_{1}\right)\left(T_{1}<T_{2}<T_{\mathrm{c}}\right)$ est plus importante que celle observée lorsque l'on redescend à $T_{1}$ soit $C_{\mathrm{p}_{2}}=\Delta W_{2} /\left(T_{2}-T_{1}\right)$ ou celle mesurée lorsque l'on remonte à $T_{2}: C_{\mathrm{p}_{3}}=\Delta W_{3} /\left(T_{2}-T_{1}\right)$.

La valeur de $C_{\mathrm{p}}$ obtenue dans les deux derniers cas (et aussi lors des cycles suivants) est toujours identique et peut être appelée chaleur spécifique réversible $C_{\mathrm{pR}}$ du matériau pour l'intervalle de température $\left(T_{1}, T_{2}\right)$. La différence $\left(C_{\mathrm{p}_{1}}-C_{\mathrm{pR}}\right) \Delta T$ semble correspondre à une chaleur latente qui n'est pas restituée lors des cycles en raison de l'hystérésis thermique de la transition (environ $10^{\circ} \mathrm{C}$ ).

Ainsi pour le titanate de plomb (Fig. 2) on peut constater que $C_{\mathrm{p}_{1}}$ atteint $0,1 \mathrm{cal} / \mathrm{g}{ }^{\circ} \mathrm{C}$ à la température de transition $T_{\mathrm{c}}=488^{\circ} \mathrm{C}$ (montée de température) alors que $C_{\mathrm{pR}}$ n'excède pas $0,14 \mathrm{cal} / \mathrm{g}{ }^{\circ} \mathrm{C}$. Par contre en dessus de $T_{\mathrm{c}}$ on a $C_{\mathrm{p} 1}=C_{\mathrm{pR}}=0,1 \mathrm{cal} / \mathrm{g}{ }^{\circ} \mathrm{C}$. On peut évaluer

$$
L=\int_{0}^{T \mathrm{c}}\left(C_{\mathrm{p}_{1}}-C_{\mathrm{pR}}\right) \mathrm{d} T=360 \mathrm{cal} / \mathrm{mole}
$$

qui correspond à la surface $S_{1}$ et

$$
\Delta H=\int_{0}^{T \mathrm{c}} \Delta C_{\mathrm{p}} \mathrm{d} T=500 \mathrm{cal} / \mathrm{mole}
$$

surface $S_{1}+S_{2}$.

$\Delta C_{\mathrm{p}}$ représentant l'anomalie totale de $C_{\mathrm{p}}$ par rapport aux valeurs obtenues en dessous de $430^{\circ} \mathrm{C}$ dans la phase antiferroélectrique. Ce résultat concorde avec ceux obtenus précédemment [3], [7] au moyen d'appareils d'analyse thermique différentielle.

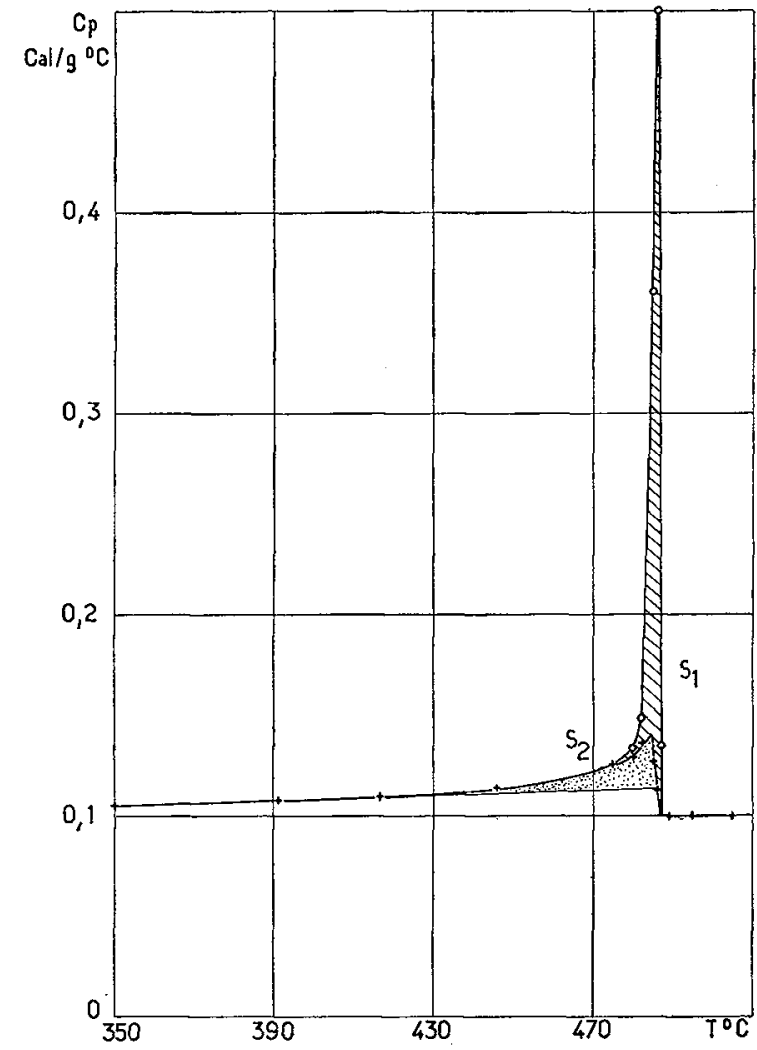

FIG. 2. - Chaleur spécifique de $\mathrm{PbTiO}_{3}$ céramique. Courbe + chaleur spécifique réversible $C_{p R}$. Courbe 0 chaleur spécifique obtenue à la première montée : $S_{1}$ aire associée à $L ; S_{1}+S_{2}$ aire associée à $\Delta H$.

La température de transition en descente de température est $T_{c}^{\prime}=479^{\circ} \mathrm{C}$ et des phénomènes analogues sont observables.

\title{
SPECIFIC HEAT ANOMALY IN THE NEIGHBOURHOOD OF A FERRO-PARAELECTRIC TRANSITIONS
}

\begin{abstract}
We report our measurements of specific heat variations versus temperature in the neighbourhood of ferro-paraelectric transitions. Two typical exemples have been chosen : T. G. S. for second order transition and $\mathrm{PbTiO}_{3}$ for first order transition. The occurrence of a thermal hysteresis in the second case is used to evaluate the latent heat associated with the transition.
\end{abstract}

It is known that when you raise the temperature through a ferro-paraelectric or an antiferro-paraelectric transition an important endothermic effect can be detected [1], [2]. This effect, which becomes exothermic when you lower temperatures, spreads over a range which can vary from a few degrees to several tens of degrees according to the nature of the material under study. Contributing to this phenomenon among others are : the thermal effect which goes together with the off-set or the on-set of spontaneous polarization; the one resulting from contraction or dilatation of the unit cell; the one resulting form the appearance or the disappearance of a domain structure etc... [3], [4].

One of the most important problems which still remains to be solved concerning these transitions is to know if, from a thermodynamic point of view, they can be considered as being of the first or the second order. In other words : is the above-said thermal effect only due to a gradual change in the specific heat, or is there a latent heat associated with these transitions ? The answer to that question would be easy to find if the material under study were monocrystals 